\title{
PLASTIC BIFURCATION AND POSTBIFURCATION ANALYSIS FOR GENERALIZED STANDARD CONTINUA
}

\author{
Son Q. Nguyen $\dagger$ and Nicolas Triantafyllidis $\ddagger$ \\ †Laboratoire de Mécanique des Solides, Ecole Polytechnique, Palaiseau 91128, France and \\ $\ddagger$ Aerospace Engineering Department, University of Michigan, Ann Arbor, MI 48109, U.S.A.
}

(Received 29 April 1988)

\begin{abstract}
THE PRESENT work is concerned with the bifurcation and postbifurcation analysis of a class of rate independent plasticity models obeying Hill's maximum dissipation principle. A variational inequality approach, which differs from the classical formulation of the plastic bifurcation problem. is employed. The rate $n$ bifurcation problem is formulated and sufficient conditions for uniqueness of the corresponding boundary value problem are given. $A$ connection is made with Hill's nonbifurcation criterion. In addition. the issue of the postbifurcation behavior of the solid is addressed in this more general context showing the possibility of angular as well as smooth bifurcations of rate $n>1$.

Finally an example, capable of exhibiting both an angular as well as a smooth bifurcation is analysed using the general formulation derived in this work. The presentation is concluded with some comments and comparisons of the present methodology with the classical approach.
\end{abstract}

\section{INTRODUCTION}

THE PLASTIC buckling of structures is a very interesting topic in mechanics, both for its practical importance as well as for its mathematical challenge. Following some early work of Considere (1891), Von Karman (1910) and Shanley (1947). Hill $(1956,1958)$ was the first to put the bifurcation instability problem for a rate independent elastoplastic continuum on a sound mathematical basis.

More specifically $\mathrm{Hil}$ has addressed the issue of loss of uniqueness in the velocities (i.e. displacement increments) for the incremental boundary value problem of an elastoplastic solid and gave sufficient conditions for the exclusion of such a bifurcation (termed rate one or angular bifurcation in the present work). He then went on to generalize the validity of his criterion by postulating the absence of a bifurcated branch at a load where a bifurcation in velocity was excluded. Subsequently, and based on Hill's formulation of the problem, HUTCHINSON $(1973,1974)$ has studied in considerable generality the postbifurcation and imperfection sensitivity issues for the aforementioned (angular) bifurcation problem. His approach is restricted to problems with total loading throughout their principal solution. In addition, fractional asymptotic expansions (in terms of the time-like parameter) of the various field quantities are required, in contrast to the (now classical) postbifurcation analysis for elastic 
systems introduced by KOITER (1945) (on this subject see also BUDIANSKY's. 1974. comprehensive review article and references quoted therein).

The present work is an outgrowth of some initially independent efforts by the authors to circumvent the problems of the plastic bifurcation and postbifurcation theory mentioned above. A mathematically simpler approach to the plastic bifurcation problem (which is also applicable to a wide range of rate independent dissipative phenomena, besides plastic stability) has recently been proposed by NGUYEN (1987) and is based on the generalized standard material formalism for rate independent solids, a concept initially introduced by HALPHEN and NGUYEN (1975). The issue of higher order (smooth) bifurcations was initially addressed by TRIANTAFYLLIDIS (1983) using a somewhat restrictive approach based on a class of CHRISTOFFERSEN and HuTCHINSON (1979) type constitutive models (i.e. models with a regularized dependence of the incremental moduli on the stress or strain rates). The work presented here can be viewed as the logical continuation of the aforementioned efforts and has the following features.

The incremental boundary value problem of rate $n$ (with respect to the time-like parameter) is formulated and sufficient conditions for its uniqueness are explored (the present results generalize some preliminary ones obtained by NGUYEN and STOLZ, 1985). In addition, a connection is made between an exclusion principle for bifurcation of arbitrary rate and Hill's exclusion of bifurcation principle, which is shown to preclude bifurcation of any order (and not merely the first rate as initially proved by Hill). The presentation continues with the formulation of the postbifurcation expansion problem in a unified approach (and in terms of an integral power series with respect to the time-like parameter) that is valid for bifurcations at any rate. The general theory is followed by a thoroughly analysed example in which both angular (rate one) and smooth (higher than rate one) bifurcations occur. Finally some concluding remarks are made about the advantages and limitations of the proposed approach.

\section{General Formulation of the Bifurcation and Postbifurcation Problem}

The generalized standard material formalism introduced by NGUYEN (1973) (see also HALPHEN and NGUYEN, 1975) will be the basis for the stability analysis presented here. Accordingly, if $\phi(\varepsilon, \alpha)$ is the free energy density function of the solid at a material point with strain $\varepsilon$ and internal variables $\alpha$, the associated stess $\mathbf{s}$ and internal forces A are given by

$$
\mathbf{s}=\frac{\partial \phi}{\partial \boldsymbol{q}}, \quad \mathbf{A}=-\frac{\partial \phi}{\partial \boldsymbol{\alpha}} .
$$

In addition, the internal forces $\mathbf{A}$ are assumed to lie inside or on the surface of a convex set $C$ defined by $f(A) \leqslant 0$. The cvolution law for the internal variables is then found from the maximum dissipation principle 


$$
\left(\mathbf{A}-\mathbf{A}^{*}\right) \cdot \dot{\alpha} \geqslant 0 \div \forall \in \mathbf{A}^{*} \in C, \quad C \equiv\left\{\mathbf{A}^{*} \mid f\left(\mathbf{A}^{*}\right) \leqslant 0\right\}
$$

where a quantity surmounted by a dot $(\cdot)$ denotes differentiation with respect to the monotonically increasing time-like parameter $\tau$.

The majority of the commonly employed small strain, rate independent plasticity laws can be cast in the above formalism. The Lagrangian strain $\varepsilon=\varepsilon(\mathbf{u})$ is a nonlinear operator of the displacement field $\mathbf{u}$ while $\mathbf{s}$ is the second Piola-Kirchhoff stress and $\phi$ is the material's free energy per unit reference volume.

From (2.2) and assuming that $f(\mathbf{A})$ is adequately smooth, one obtains

$$
\dot{\alpha}=\mu \frac{\partial f}{\partial \mathbf{A}} \text { with }\left\{\begin{array}{l}
\mu=0 \text { if } f(\mathbf{A})<0 \\
\mu \geqslant 0 \text { if } f(\mathbf{A})=0 .
\end{array}\right.
$$

The constitutive equations (2.1), (2.2) (or (2.1) and (2.3)) will be subsequently employed in the formulation of the boundary value problem for the rate independent elastoplastic materials considered in this work.

In addition to (2.1)-(2.3) the following useful relations are recorded: by subsequently taking $\mathrm{A}^{*}$ to be $\mathbf{A}(\tau) \pm \dot{\mathbf{A}}(\tau) \Delta \tau$ and assuming a smooth dependence of $f$ on $A$, one deduces from (2.2)

$$
\dot{\mathbf{A}} \cdot \dot{\alpha}=0 \Rightarrow \mu \frac{\partial f}{\partial \mathbf{A}} \cdot \dot{\mathbf{A}}=\mu \dot{f}=0 .
$$

The $n$ rates of (2.4) as well as (2.1)-(2.3) will be required for the determination of the solution to the boundary value problem of rate $n$.

This section is only meant as a brief reminder for the governing equations of the generalized standard model. For a more detailed account of the theory accompanied by appropriate illustrative examples, the interested reader is referred to NGUYEN (1980).

\subsection{Uniqueness for the boundary value problem of rate $\mathrm{n}$}

Consider a generalized standard solid with free energy $\phi(\varepsilon, \boldsymbol{x})$ per unit reference volume occupying a volume $\Omega$ with boundary $\partial \Omega$. Moreover assume that on a part of the boundary, say $\partial \Omega_{u}$ the displacements $\mathbf{u}$ are prescribed while on the remaining part of the boundary, say $\partial \Omega_{l}$, surface tractions $\lambda \mathbf{T}^{0}$ are applied in proportion to a scalar parameter $\lambda$. Neglecting body forces for simplicity and assuming $U$ to be the space of all admissible displacement functions u, the solid's potential energy is given by :

$$
\mathscr{E}=\int_{\Omega} \varphi(\varepsilon, \boldsymbol{\alpha}) \mathrm{d} V-\lambda \int_{i \Omega} \mathbf{T}^{0} \cdot \mathbf{u} \mathrm{d} S
$$

where for simplicity it has been assumed that $\mathbf{u}=0$ on $\partial \Omega_{t}$.

$\dagger$ Note: For notational convenience the inner product between tensors of arbitrary order will be denoted by $(\cdot)$. 
The equilibrium equation, i.e. the boundary value problem of rate 0 , is given by extremizing $\mathscr{E}$ with respect to u. Thus,

$$
\mathscr{E}_{, \mu}(\delta \mathbf{u})=\int_{\Omega}\left[\frac{\partial \phi}{\partial \boldsymbol{\varepsilon}} \cdot \delta \varepsilon\right] \mathrm{d} V-\lambda \int_{\hat{\Omega} \Omega} \mathbf{T}^{0} \cdot \delta \mathbf{u} \mathrm{d} S=0
$$

where $\delta \boldsymbol{\varepsilon}=\boldsymbol{\varepsilon}_{u}(\delta \mathbf{u})$ is the first variation of the (nonlinear) strain-displacement operator $\boldsymbol{\varepsilon}(\mathbf{u})$ with respect to $\mathbf{u}$. Assuming at this stage that the displacement $\mathbf{u}$ (and hence the strain $\varepsilon$ ) and the internal variable $\alpha$ fields are known at an instant $\tau$. one is interested in determining the rates of the aforementioned fields $\dot{\mathbf{u}}$ and $\dot{\alpha}$, or equivalently from (2.3) $\dot{\mathbf{u}}$ and $\mu$. It will be shown that $(\dot{\mathbf{u}}, \mu)$ is the solution to the following variational inequality:

$$
\begin{aligned}
& \int_{\Omega}\left[\left(\frac{\partial \phi}{\partial \boldsymbol{\varepsilon}} \cdot \delta \varepsilon\right)^{\cdot}+(\beta-\mu)(-\dot{f})\right] \mathrm{d} V-\dot{\lambda} \int_{c \Omega} \mathbf{T}^{0} \cdot \delta \mathbf{u} \mathrm{d} S \geqslant 0 \quad \forall(\delta \mathbf{u}, \beta) \in U \times K_{1}, \\
& K_{1} \equiv\left\{\beta(\mathbf{x}) \mid \beta \geqslant 0 \text { if } \mathbf{x} \in I_{1}^{0}, \quad \beta=0 \text { if } \mathbf{x} \in I_{1}^{-}\right\}, \\
& I_{1}^{+} \equiv \varnothing, \\
& I_{1}^{0} \equiv\{\mathbf{x} \in \Omega \mid f(\mathbf{A}(\mathbf{x}))=0\}, \\
& I_{1}^{-} \equiv\{\mathbf{x} \in \Omega \mid f(\mathbf{A}(\mathbf{x}))<0\} .
\end{aligned}
$$

Note that by definition the sets $I_{1}^{1}, I_{1}^{0}, I_{1}^{-}$are disjoint and that their union gives $\Omega$. i.e. $I_{1}^{+} \cup I_{1}^{0} \cup I_{1}^{-}=\Omega$.

At this point it is tactitly assumed that no moving discontinuities of the quantity $(\partial \phi / \partial \varepsilon) \cdot \delta \varepsilon$ exist. Consequently, the first rate of the equilibrium Eq. (2.6), when written out explicitly, does not contain any surface terms. This assumption is satisfied in most applications of interest for it corresponds to problems with continuous stress (see (2.1)) and strain fields or more generally to problems with stationary stress and strain discontinuities. This assumption is no longer valid for the rates of the aforementioned quantities, as will be discussed later.

By taking $\delta \mathbf{u} \neq 0$ and $\beta=\mu$, one recovers the first rate of the equilibrium Eq. (2.6). Conversely, for $\delta \mathbf{u}=0, \beta \neq \mu$ and noting that $\beta, \mu \in K_{1}$ one has

$$
\begin{aligned}
\int_{\Omega}(\beta-\mu)(-\dot{f}) \mathrm{d} V \geqslant 0 \Rightarrow \int_{l_{i}^{+} \cup l_{1}^{0}}(\beta-\mu)(-\dot{f}) \mathrm{d} V & \geqslant 0 \\
& \Rightarrow \text { For } \mathbf{x} \in I_{1}^{0}\left\{\begin{array}{l}
\dot{f} \leqslant 0 \text { if } \mu=0 \\
\dot{f}=0 \text { if } \mu>0,
\end{array}\right.
\end{aligned}
$$

exactly as predicted from (2.3) and (2.4).

Having formulated the rate 1 boundary value problem, attention is focused on the uniqueness of its solution $(\dot{\mathbf{u}}, \mu)$. To this cnd, consider the following functional $\mathscr{F}[\hat{\mathbf{u}}, \beta]$, quadratic in $(\hat{\mathbf{u}}, \beta)$, defined by:

$$
\begin{aligned}
\mathscr{F}[\hat{\mathbf{u}}, \beta] \equiv & \int_{\Omega}\left[\boldsymbol{\varepsilon}_{{ }_{u}}(\hat{\mathbf{u}}) \cdot \frac{\partial^{2} \phi}{\partial \boldsymbol{\varepsilon} \hat{\partial} \boldsymbol{\varepsilon}} \cdot \boldsymbol{\varepsilon}_{{ }_{u}}(\hat{\mathbf{u}})+\boldsymbol{\varepsilon}_{u_{u}}(\hat{\mathbf{u}}) \cdot \frac{\partial^{2} \phi}{\partial \boldsymbol{\varepsilon} \partial \boldsymbol{\alpha}} \cdot \frac{\partial f}{\partial \mathbf{A}} \beta\right. \\
& \left.+\beta \frac{\partial f}{\partial \mathbf{A}} \cdot \frac{\partial^{2} \phi}{\partial \boldsymbol{\alpha} \partial \boldsymbol{\varepsilon}} \cdot \boldsymbol{\varepsilon}_{,_{u}}(\hat{\mathbf{u}})+\beta \frac{\partial f}{\partial \mathbf{A}} \cdot \frac{\partial^{2} \phi}{\partial \alpha \partial \boldsymbol{\partial} \boldsymbol{\partial}} \cdot \frac{\partial f}{\partial \mathbf{A}} \beta+\frac{\partial \phi}{\partial \boldsymbol{\varepsilon}} \cdot \boldsymbol{\varepsilon}_{,_{u u}}(\hat{\mathbf{u}}, \hat{\mathbf{u}})\right] \mathrm{d} V .
\end{aligned}
$$


If $\mathscr{F}[\hat{\mathbf{u}}, \beta]$ is positive definite $\forall(\hat{\mathbf{u}}, \beta) \in U \times V K_{1}$ (with $V K$ denoting the linear hull of the convex set $K$ ), it will be shown that a rate 1 bifurcation is excluded. For if not, assume $\left(\dot{\mathbf{u}}_{1}, \mu_{1}\right)$ and $\left(\dot{\mathbf{u}}_{2}, \mu_{2}\right)$ to be two different solutions of the variational inequality in (2.7). Employing (2.7) and (2.8) in (2.9) one obtains the contradiction

$$
\mathscr{F}\left[\dot{\mathbf{u}}_{1}-\dot{\mathbf{u}}_{2}, \mu_{1}-\mu_{2}\right]=\int_{I_{1}^{0}}\left(\mu_{1}-\mu_{2}\right)\left(-\dot{f}_{1}+\dot{f}_{2}\right) \mathrm{d} V \leqslant 0 .
$$

The positive definiteness of $\mathscr{F}$ on $U \times V K_{1}$ is a sufficient condition for the uniqueness of the rate 1 problem and hence is too restrictive. One expects a unique solution to the rate 1 problem in (2.7) under less stringent conditions.

Once a unique solution to $(2.7)$ is found, i.e. given $(\mathbf{u}, \boldsymbol{\alpha}),(\dot{\mathbf{u}}, \dot{\alpha})$, the rate 2 problem can be formulated. It will be shown that $(\ddot{\mathbf{u}}, \dot{\mu})$ is the solution to the variational inequality:

$$
\begin{aligned}
& \int_{\Omega}\left[\left(\frac{\partial \phi}{\partial \varepsilon} \cdot \delta \varepsilon\right)^{*}+(\beta-\mu)(-\ddot{f})\right] \mathrm{d} V-\ddot{\gamma} \int_{c \Omega} \mathbf{T}^{0} \cdot \delta \mathbf{u} \mathrm{d} S \geqslant 0 \quad \forall(\delta \mathbf{u}, \beta) \in U \times K_{2}, \\
& K_{2} \equiv\left\{\beta(\mathbf{x}) \mid \beta \in R \text { if } \mathbf{x} \in I_{1}^{+} \cup I_{2}^{+}, \quad \beta \geqslant 0 \text { if } \mathbf{x} \in I_{2}^{0}, \quad \beta=0 \text { if } \mathbf{x} \in I_{2}^{-} \cup I_{1}^{-}\right\}, \\
& I_{2}^{+} \equiv\left\{\mathbf{x} \in I_{1}^{0} \mid \mu(\mathbf{x})>0, \quad \dot{f}(\mathbf{A}(\mathbf{x}))=0\right\}, \\
& I_{2}^{0} \equiv\left\{\mathbf{x} \in I_{1}^{0} \mid \mu(\mathbf{x})=0, \quad \dot{f}(\mathbf{A}(\mathbf{x}))=0\right\}, \\
& I_{2}^{-} \equiv\left\{\mathbf{x} \in I_{1}^{0} \mid \mu(\mathbf{x})=0, \quad \dot{f}(\mathbf{A}(\mathbf{x}))<0\right\} .
\end{aligned}
$$

Note also that the sets $I_{2}^{+}, I_{2}^{0}, I_{2}^{-}$are disjoint and that their union gives $I_{1}^{0}$, i.e. $I_{2}^{+} \cup I_{2}^{0} \cup I_{2}^{-}=I_{1}^{0}$.

Unlike $(\partial \phi / \partial \varepsilon) \cdot \delta \boldsymbol{\varepsilon}$ its rate 1 derivative with respect to $\tau$ can have moving discontinuities. Hence the term $\left((\partial \phi / \partial \varepsilon \cdot \delta \varepsilon){ }^{*}\right.$ in $(2.11)$ has to be interpreted in the generalized function sense in order to account for the time-dependent discontinuities in this quantity. Had a more explicit notation been adopted, some additional surface integrals would have also been included in (2.11). Knowing that the discontinuity in the rate of a field quantity, say $\dot{g}$, propagating with velocity $v_{1}$ in the direction of the outward normal $\mathbf{n}$ to the discontinuity surface in question satisfies $(\llbracket g \nabla \rrbracket \cdot \mathbf{n}) v_{1}+\llbracket \dot{g} \rrbracket=0$, one deduces that the surface discontinuity terms in (2.11) involve field quantities up to rate one. This remark is important when considering bifurcation of the rate 2 problem for only the volume term given in (2.9) appears in the final calculation.

Similarly to the rate 1 case, by taking $\delta \mathbf{u} \neq 0, \beta=\dot{\mu}$ one recovers the second rate of the equilibrium equation (2.6). Moreover, for $\delta \mathbf{u}=0, \beta \neq \dot{\mu}$ and since $\beta, \dot{\mu} \in K_{2}$ (2.11) yields :

$$
\int_{\Omega}(\beta-\ddot{\mu})(-\ddot{f}) \mathrm{d} V \geqslant 0 \Rightarrow \int_{l_{1}^{+} \cup I^{\ddagger} \cup I_{2}^{0}}(\beta-\ddot{\mu})(-\ddot{f}) \mathrm{d} V \geqslant 0
$$

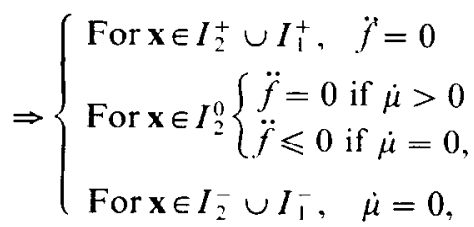


which can be easily verified by taking rates of (2.2)-(2.4). Notice also that for the rate 2 problem $\ddot{\mu} \ddot{f}=0 \quad \forall \mathbf{x} \in \Omega$ (compare with (2.4)).

The natural question arising next pertains to the uniqueness of the solution $(\ddot{\mathbf{u}}, \dot{\mu})$ to the rate 2 boundary value problem. It will be shown that a sufficient condition for the uniqueness of a solution to (2.11) is the positive definiteness $\mathscr{F}[\hat{\mathbf{u}}, \beta]$ on $U \times V K_{2}$. Considering again $\mathscr{F}\left[\ddot{\mathbf{u}}_{1},-\ddot{\mathbf{u}}_{2}, \dot{\mu}_{1},-\dot{\mu}_{2}\right]$ for the two different solutions $\left(\ddot{\mathbf{u}}_{1}, \dot{\mu}_{1}\right)$ and $\left(\ddot{\mathbf{u}}_{2}, \dot{\mu}_{2}\right)$ to the rate 2 problem (2.11) (with the tacit assumption that up to the time of interest the solutions to the rate 0 and 1 problems are unique) and making use of (2.9). (2.11) and (2.12) one has

$$
\mathscr{F}\left[\ddot{\mathbf{u}}_{1}-\ddot{\mathbf{u}}_{2}, \dot{\mu}_{1}-\dot{\mu}_{2}\right]=\int_{I_{2}^{0}}\left(\dot{\mu}_{1}-\dot{\mu}_{2}\right)\left(-\ddot{f}_{1}+\ddot{f}_{2}\right) \leqslant 0,
$$

which obviously contradicts the assumption about the positive definiteness of $\overline{\mathscr{F}}$.

The rate 3 problem, which will be examined next, suggests the algorithm for the generalization to the problem of rate $n$. Once more, it is assumed that a unique solution has been found to the problems of rates 0,1 and 2 up to the time of interest. The wanted solution $(\ddot{\mathbf{u}}, \ddot{\mu})$ is determined from the following variational inequality:

$$
\begin{aligned}
& \int_{\Omega}\left[\left(\frac{\partial \phi}{\partial \varepsilon} \cdot \delta \varepsilon\right)^{\cdots}+(\beta-\ddot{\mu})(-\bar{f})\right] \mathrm{d} V-\bar{\lambda} \int_{\partial \Omega} \mathbf{T}^{0} \cdot \delta \mathbf{u} \mathrm{d} S \geqslant 0 \quad \forall(\delta \mathbf{u}, \beta) \in U \times K_{3}, \\
& K_{3} \equiv\left\{\beta(\mathbf{x}) \mid \beta \in R \text { if } x \in I_{1}^{+} \cup I_{2}^{+} \cup I_{3}^{+}, \quad \beta \geqslant 0 \text { if } \mathbf{x} \in I_{3}^{0},\right. \\
& \left.\quad \beta=0 \text { if } \mathbf{x} \in I_{3}^{-} \cup I_{2}^{-} \cup I_{1}^{-}\right\}, \\
& I_{3}^{+} \equiv\left\{\mathbf{x} \in I_{2}^{0} \mid \dot{\mu}(\mathbf{x})>0, \quad \ddot{f}(\mathbf{A}(\mathbf{x}))=0\right\}, \\
& I_{3}^{0} \equiv\left\{\mathbf{x} \in I_{2}^{0} \mid \dot{\mu}(\mathbf{x})=0, \quad \ddot{f}(\mathbf{A}(\mathbf{x}))=0\right\}, \\
& I_{3}^{-} \equiv\left\{\mathbf{x} \in I_{2}^{0} \mid \dot{\mu}(\mathbf{x})=0, \quad \ddot{f}(\mathbf{A}(\mathbf{x}))<0\right\} .
\end{aligned}
$$

As in (2.7) and (2.11) by construction $I_{3}^{+}, I_{3}^{0}, I_{3}^{-}$are disjoint with $I_{3}^{+} \cup I_{3}^{0} \cup I_{3}^{-}=I_{2}^{0}$.

Similarly to the rate 2 problem, the rate 3 derivative of $(\partial \phi / \partial \varepsilon) \cdot \delta \varepsilon$ with respect to $\tau$ appearing in (2.14) has to be interpreted in the generalized function sense in view of the possible moving discontinuities. In writing (2.14) explicitly, only the volume integral contains rate 3 terms while the surface discontinuity integrals contain terms up to rate two. Hence in the bifurcation calculation of the rate 3 problem only the volume term in (2.9) appears in the final expression.

Once more the third rate of the equilibrium equation (2.6) is recovered from (2.14) by taking $\delta \mathbf{u} \neq 0, \beta=\ddot{\mu}$, while for $\delta \mathbf{u}=0, \beta \neq \ddot{\mu}$ and given that $\beta, \ddot{\mu} \in K_{3}$ one obtains

$$
\begin{aligned}
& \int_{\Omega}(\beta-\ddot{\mu})(-\bar{f}) \mathrm{d} V \geqslant 0 \Rightarrow \int_{l^{\uparrow} \cup \ell^{\ddagger} \cup l^{\ddagger} \cup I_{3}^{0}}(\beta-\ddot{\mu})(-\bar{f}) \geqslant 0
\end{aligned}
$$

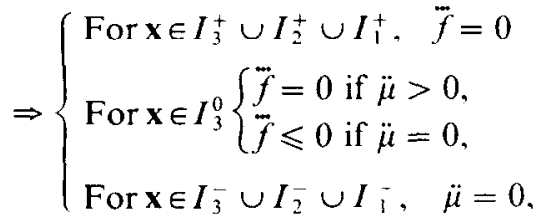


which also follows from (2.2), (2.3) and (2.4). As for the previous two cases, one has from (2.15) $\ddot{\mu} \vec{f}=0 \quad \forall \mathbf{x} \in \Omega$.

As expected, a sufficient condition for the uniqueness of the solution ( $\tilde{\mathbf{u}}, \ddot{\mu})$ to (2.14) is the positive definiteness of the functional $\mathscr{F}$ in (2.9) over the set $U \times V K_{3}$. Indeed, by a similar construction, if $\left(\tilde{\mathbf{u}}_{1}, \ddot{\mu}_{1}\right)$ and $\left(\ddot{\mathbf{u}}_{2}, \ddot{\mu}_{2}\right)$ are two different solutions to (2.14), from (2.9), (2.14) and (2.15) follows the contradiction

$$
\mathscr{F}\left[\overline{\mathbf{u}}_{1}-\overline{\mathbf{u}}_{2}, \ddot{\mu}_{1}-\ddot{\mu}_{2}\right]=\int_{I_{3}^{0}}\left(\ddot{\mu}_{1}-\ddot{\mu}_{2}\right)\left(-\bar{f}_{1}+\bar{f}_{2}\right) \leqslant 0 .
$$

The generalization of the above algorithm for the rate $n$ boundary value problem can be accomplished as follows: assuming a unique response $(\dot{\mathbf{u}}, \mu),(\ddot{\mathbf{u}}, \dot{\mu}), \ldots$, $\left({ }^{(n-1)},{ }^{(n-2)} \mu^{2}\right)$ to all the boundary value problems of order $1,2, \ldots(n-1)$, the variational inequality corresponding to the rate $n$ problem is given by $\dagger$

$$
\begin{aligned}
& \int_{\Omega}\left[\left(\frac{\partial \phi}{\partial \varepsilon} \cdot \delta \varepsilon\right)^{(n)}+\left(\beta-\stackrel{(n-1)}{\mu}^{\prime}\right)(-\stackrel{(n)}{f})\right] \mathrm{d} V-\stackrel{(n)}{\lambda} \int_{a \Omega} \mathbf{T}^{0} \cdot \delta \mathbf{u} \mathrm{d} S \geqslant 0 \quad \forall(\delta \mathbf{u}, \beta) \in U \times K_{n}, \\
& K_{n} \equiv\left\{\beta(\mathbf{x}) \mid \beta \in R \text { if } \mathbf{x} \in \bigcup_{k=1}^{n} I_{k}^{+}, \quad \beta \geqslant 0 \text { if } \mathbf{x} \in I_{n}^{0}, \quad \beta=0 \text { if } \mathbf{x} \in \bigcup_{k=1}^{n} I_{k}^{-}\right\}, \\
& I_{n}^{+} \equiv\left\{\left.\mathbf{x} \in I_{n-1}^{0}\right|^{(n-2)} \mu(\mathbf{x})>0, \quad{ }^{(n-1)}(\mathbf{A}(\mathbf{x}))=0\right\},
\end{aligned}
$$

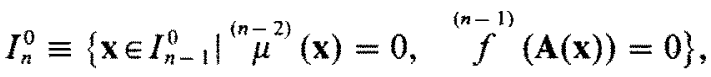

$$
\begin{aligned}
& I_{n}^{-} \equiv\left\{\left.\mathbf{x} \in I_{n-1}^{0}\right|^{(n-2)} \mu(\mathbf{x})=0, \quad \stackrel{(n-1)}{f}(\mathbf{A}(\mathbf{x}))<0\right\} .
\end{aligned}
$$

Note that $I_{n}^{+}, I_{n}^{0}, I_{n}^{-}$are disjoint while $I_{n}^{+} \cup I_{n}^{0} \cup I_{n}^{-}=I_{n-1}^{0}$.

As in all the previous cases for $\delta \mathrm{u} \neq 0, \beta={ }^{(n-1)}{ }^{2}$ the variational inequality (2.17) yields the equilibrium equation (2.6) of rate $n$ while for $\delta \mathbf{u}=0, \beta \neq{ }^{(n-1)} \mu$ and since $\beta$, $\stackrel{(n-1)}{\mu} \in K_{n}$

†Note: Of special interest for the uniqueness argument is the term of (2.17), that contains the highest order time derivatives $(n)$ and ${ }^{(n-1)} \mu$, which is

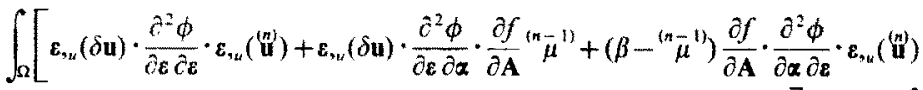

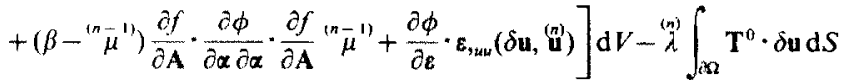

$$
\begin{aligned}
& +O\left({ }^{(n-1)} \cdot\left(n-\mu^{2)}\right)\right. \text { terms. }
\end{aligned}
$$

It is interesting to note that the surface discontinuity terms in the explicitly written out form of $(2.17)$, are at most of $O\left({ }^{(n} \bar{u}^{1)},{ }^{(n-2)}\right)$. This property follows from the observation that the normal speed of propagation $v_{m}$ of a surface discontınuity of the rate $m$ in tield quantity $g$ is given by $\left(\left[^{(m-g} g^{1)} \nabla \rrbracket \cdot n\right) v_{m}+\llbracket\left[g^{\prime \prime}\right) \rrbracket=0\right.$ where $\mathbf{n}$ is the outward surface normal in the direction of propagation of the discontinuity jump [ $]$. 
$\int_{\Omega}\left(\beta-{ }^{(n-1)} \mu^{1)}\right)(-\stackrel{(n)}{f}) \geqslant 0 \Rightarrow \int\left({\left.\stackrel{n}{=} I_{i}\right) \cup I_{n}^{(n)}}\left(\beta-\stackrel{(n-1)}{\mu}^{\prime \prime}\right)(-\stackrel{(n)}{f}) \geqslant 0\right.$

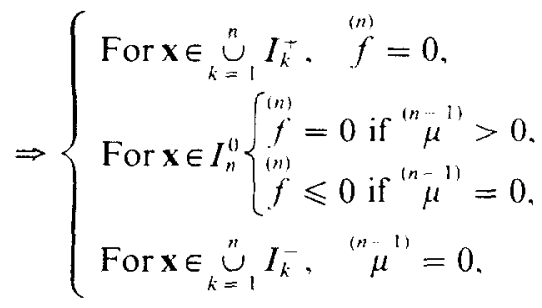

which similarly follows from (2.2), (2.3) and (2.4) after taking appropriate rates. Also note that $\stackrel{(n-1)^{(n)}}{f}=0 \quad \forall \mathbf{x} \in \Omega$.

A sufficient condition for the uniqueness of the solution $\left(\stackrel{(n)}{(\mathbf{u},},{ }^{(n-1)}\right)$ to $(2.17)$ is again the positive definiteness of the functional $\mathscr{F}$ defined in (2.9) over the set $U \times V K_{n}$. For if not, assuming the existence of two different solutions $\left(\mathbf{u}_{1},{ }^{(n-1)} \mu_{1}^{1)}\right),\left(\mathbf{u}_{2},{ }^{(n)} \mu_{2}\right)$ and making use of (2.9), (2.17) and (2.18) one is lead to the contradiction

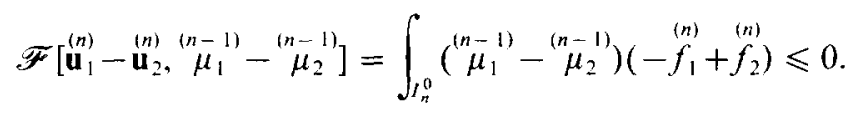

Note that in the derivation of (2.19) use was made of the previous remark that only the volume part in the incremental equilibrium equation of rate $n$ contains terms of order $n$. All surface discontinuity terms are of rate $n-1$ or lower and hence do not appear in (2.19).

The development of the variational inequality method for the rate problem of order $n$ is a generalization of the recent work by NGUYEN and STOLz (1985) for the problems of rates 1 and 2. Note that in contrast to the aforementioned work, in the present development no explicit expression for the $n$th rate of the virtual work is given (see Eqs $(2.7),(2.11), 2.14),(2.17)$ ) in view of the resulting cumbersome expressions. It should also be repeated at this point that special care has to be taken when considering the explicit expressions for the rates of the equilibrium Eq (2.6) in view of the surfaces of discontinuity in ${ }^{(n-1)} \boldsymbol{\varepsilon}$ and ${ }^{(n-1)} \alpha^{2}$; this difficulty will become apparent in the next subsection as well as in the example. The important point to retain is that for the reasons given in the discussion note for $(2.17)$, the surface discontinuity terms involve rates of order $n-1$ and lower, a key property required in the derivation of (2.19).

It is not difficult to see that $V K_{1} \supset V K_{2} \supset \ldots \supset V K_{n} \ldots$. Hence positive definiteness of $\mathscr{\mathscr { F }}[\hat{\mathbf{u}}, \beta] \quad \forall(\hat{\mathbf{u}}, \beta) \in U \times V K_{1}$ and $\forall \lambda \in\left[0, \lambda_{c}\right)$ (where $\mathscr{F}$ is evaluated on the principal solution $u(\lambda), \alpha(\lambda))$ implies the impossibility of bifurcation in any order in the load parameter interval $\left[0, \lambda_{c}\right)$. If one makes the conjecture that any possible bifurcation is amenable to a polynomial type one, i.e. that there exists a parametrization at a neighborhood of the critical load $\lambda$, such that (NGUYEN, 1987) 


$$
\begin{gathered}
\lambda(\tau)=\dot{\lambda}_{c}+\frac{\tau^{p}}{p !} \lambda_{p}+\mathrm{O}\left(\tau^{p-1}\right) \quad 0<\tau \ll 1, \mathbf{u}_{q} \neq 0, \\
\mathbf{u}(\tau)=\stackrel{0}{\mathbf{u}}(\tau)+\frac{\tau^{q}}{q !} \mathbf{u}_{q}+\mathrm{O}\left(\tau^{q+1}\right), \quad \boldsymbol{\alpha}(\tau)=\stackrel{0}{\boldsymbol{\alpha}}(\tau)+{ }_{q !}^{\tau^{q}} \boldsymbol{\alpha}_{q}+\mathrm{O}\left(\tau^{q+1}\right),
\end{gathered}
$$

where $p, q \in N \backslash\{0\}$, then the positive definiteness of $\mathscr{F}$ on $U \times V K_{1}$ for a solution $(\mathbf{u}(\lambda), \stackrel{0}{\alpha}(\lambda))$ on an interval $\left[0, \lambda_{c}\right)$ excludes bifurcation at that interval.

At this point a connection is made between Hill's sufficiency criterion for nonbifurcation and the one presently proposed. To this end, according to HiLl (1958), the following functional $\mathscr{H}[\hat{\mathbf{u}}]$ (quadratic in $\hat{\mathbf{u}}$ ) is defined :

$$
\begin{array}{r}
\mathscr{H}[\hat{\mathbf{u}}] \equiv \int_{\Omega}\left\{\boldsymbol{\varepsilon}, u(\hat{\mathbf{u}}) \cdot\left[\frac{\partial^{2} \phi}{\partial \boldsymbol{\varepsilon} \partial \boldsymbol{\varepsilon}}-\chi_{I_{1}^{0}}\left(\frac{\partial^{2} \phi}{\partial \boldsymbol{\varepsilon} \partial \boldsymbol{\alpha}} \cdot \frac{\partial f}{\partial \mathbf{A}}\right)\left(\frac{\partial f}{\partial \mathbf{A}} \cdot \frac{\partial^{2} \phi}{\partial \boldsymbol{\alpha} \partial \boldsymbol{\alpha}} \cdot \frac{\partial f}{\partial \mathbf{A}}\right)^{-1}\left(\frac{\partial f}{\partial \mathbf{A}} \cdot \frac{\partial^{2} \phi}{\partial \boldsymbol{\alpha} \partial \boldsymbol{\varepsilon}}\right)\right] \cdot \boldsymbol{\varepsilon}_{u_{u}}(\hat{\mathbf{u}})\right. \\
\left.+\frac{\partial \phi}{\partial \boldsymbol{\varepsilon}} \cdot \boldsymbol{\varepsilon}_{,_{u}}(\hat{\mathbf{u}}, \hat{\mathbf{u}})\right\} \mathrm{d} V, \quad \hat{\mathbf{u}} \in U,
\end{array}
$$

with $\chi_{I_{1}^{0}}$ the characteristic function of $I_{1}^{0}$ (i.e. $\chi(\mathbf{x})=1$ if $\mathbf{x} \in I_{1}^{0}$ and $\chi(\mathbf{x})=0$ if $\mathbf{x} \notin I_{1}^{0}$ ).

HILL (1958) has shown that positive definiteness of $\mathscr{H}$ in an interval $\left[0, \lambda_{c}\right)$ of the load parameter $\lambda$ excludes the bifurcation in the rate 1 problem there. Without addressing the question of any higher order bifurcation, he postulated that bifurcation in the interval $\left[0, \lambda_{c}\right.$ ) (where $\mathscr{H}$ is positive definite) is excluded. The result presented herc provides the justification for Hill's far sighted intuition. It will be shown that positive definiteness of $\mathscr{H}$ in $\left[0, \lambda_{c}\right.$ ) implies (assuming certain additional properties of $\mathscr{F}$ which are met in the applications of interest) the positive definiteness of $\mathscr{F}$ on the same interval and hence bifurcation in any rate is excluded.

Indeed, had this not been the case, i.e. if $\mathscr{F}$ is not positive definite at some point of $\left[0, \lambda_{r}\right)$ and given that $\mathscr{F}$ is positive definite at $\lambda=0$ (otherwise the problem is ill posed since a bifurcation is possible at a vanishing externally applied load) from the continuity of $\mathscr{F}$ with respect to $\lambda$ there exists $\lambda^{*} \in\left[0, \lambda_{c}\right)$ such that $\lambda^{*}$ is the point of the first loss of positive definiteness of $\mathscr{F}$ as $\lambda$ increases away from zero. Without loss of generality, it can also be assumed that at $\lambda=\lambda^{*}$, one can find $\left(\dot{\mathbf{u}}^{*}, \mu^{*}\right) \in U \times V K_{\text {l }}$ such that

$$
\begin{aligned}
& \int_{\Omega}\left\{\boldsymbol{\varepsilon}_{,_{u}}(\hat{\mathbf{u}}) \cdot\left[\frac{\hat{\partial}^{2} \phi}{\hat{\partial} \boldsymbol{\varepsilon} \hat{\partial} \boldsymbol{\varepsilon}} \cdot \boldsymbol{\varepsilon}_{{ }_{u}}\left(\dot{\mathbf{u}}^{*}\right)+\frac{\partial^{2} \phi}{\partial \boldsymbol{\varepsilon} \hat{\partial \boldsymbol{\alpha}}} \cdot \frac{\partial f}{\partial \mathbf{A}} \mu^{*}\right]+\beta\left[\frac{\partial f}{\partial \mathbf{A}} \cdot \frac{\partial^{2} \phi}{\partial \boldsymbol{\alpha} \partial \boldsymbol{\varepsilon}} \cdot \boldsymbol{\varepsilon}_{{ }_{u}}\left(\dot{\mathbf{u}}^{*}\right)\right.\right. \\
& \left.\left.+\frac{\partial f}{\partial \mathbf{A}} \cdot \frac{\partial^{2} \phi}{\partial \boldsymbol{\alpha} \partial \boldsymbol{\alpha}} \cdot \frac{\partial f}{\partial \mathbf{A}} \mu^{*}\right]+\frac{\partial \phi}{\partial \boldsymbol{\varepsilon}} \cdot \boldsymbol{\varepsilon}_{{ }_{u u}}\left(\hat{\mathbf{u}}, \dot{\mathbf{u}}^{*}\right)\right\} \mathrm{d} V=0, \quad \forall(\hat{\mathbf{u}}, \beta) \in U \times V K_{1} .
\end{aligned}
$$

For $\hat{\mathbf{u}}=0$ and $\beta \neq 0$, from (2.22) follows that

$$
\mu^{*}=-\chi_{l_{1}^{0}}\left(\frac{\partial f}{\partial \mathbf{A}} \cdot \frac{\partial^{2} \phi}{\partial \boldsymbol{\alpha} \partial \boldsymbol{\varepsilon}} \cdot \boldsymbol{\varepsilon}_{{ }_{u}}\left(\dot{\mathbf{u}}^{*}\right)\right)\left(\frac{\partial f}{\partial \mathbf{A}} \cdot \frac{\partial^{2} \phi}{\partial \boldsymbol{\alpha} \partial \boldsymbol{\alpha}} \cdot \frac{\partial f}{\partial \mathbf{A}}\right)^{1}
$$

since $\beta(\mathbf{x})=0 \quad \forall \mathbf{x} \in I_{1}^{-}$, for $\beta \in V K_{1}$. Substitution of (2.23) into (2.22) and after taking $\hat{\mathbf{u}}=\dot{\mathbf{u}}^{*}$, one easily obtains the contradiction that at $\lambda^{*} \in\left[0, \lambda_{c}\right), \mathscr{H}\left[\dot{\mathbf{u}}^{*}\right]=0$. 
A discussion on why Hill's uniqueness criterion excludes not only rate 1 bifurcations but higher order ones as well, was given in a different setting by TRIANTAFYLLIDIS (1983). Some more recent work by PETRYK and THERMANN (1985) has subsequently addressed the smonth bifurcation problem but only up to the second rate and by employing a completely different approach than the one proposed here.

\subsection{Postbifurcation analysis-smooth vs angular bifurcations}

Having established a sufficient condition for non-bifurcation, attention is next focused on its failure and more specifically on the postbifurcation asymptotic expansions for generalized standard solids.

Following NGUYEN (1987), a polynomial expansion in terms of integral powers of the time-like parameter $\tau$ is postulated near the critical load $\lambda_{c}$ (i.e. the load at which the positive definiteness of $\mathscr{F}$ is lost for the first time as $\lambda$ moves away from zero), as indicated by (2.20). A bifurcation is termed "angular" if $p=q^{\dagger}$ and "smooth" if $p<q$ the terminology being motivated by the geometry of the corresponding $\lambda-\|\mathbf{u}\|$ diagram where the bifurcated branch emerges at an angle $\left(\lim _{\lambda \rightarrow i_{c}}\|\mathbf{u}-\mathbf{u}\| /\left(\lambda-\lambda_{c}\right) \neq 0\right)$ or tangentially $\left(\lim _{\lambda \rightarrow i_{c}}\|\mathbf{u}-\stackrel{0}{\mathbf{u}}\| /\left(\lambda-\lambda_{c}\right)=0\right.$ ) from the principal branch $\mathbf{u}(\lambda)$.

Unlike the postbifurcation analysis of elastic solids where (in general) adequate regularity of the postbifurcation solution is available and permits a straightforward and general scheme for the calculation of all the higher order terms as found by KOITER (1945) (see also BUDIANSKY's, 1974 comprehensive review article), no such regularity is present even in the simplest meaningful problems in plasticity in view of the presence of propagating discontinuities in $\stackrel{(n)}{\alpha}$. Hence only the first terms (i.e. $\left.\lambda_{p}, \mathbf{u}_{q}, \boldsymbol{\alpha}_{q}\right)$ in the postbifurcation expansions in $(2.20)$ will be sought via the general method proposed here.

It is not difficult to deduce from (2.20) that $I_{1}^{0}=I_{2}^{0}=\ldots I_{p}^{0}$. Indeed, by assuming (without loss of generality) $(\mathrm{d} \mathbf{u} / \mathrm{d} \lambda)_{(w,-1)} \neq 0$, one has $\dot{\mathbf{u}}=\ddot{\mathbf{u}}=\ldots{ }^{(p-1)} \mathbf{u}^{-1)}=0$ and hence $\mu=\dot{\mu}=\ldots \mu^{(p)}=0, f=f=\ddot{f}=\ldots f^{(p-1)}=0$ on $I_{p}^{0}=I_{1}^{0}$. At bifurcation noting that $\left(\mathbf{u}_{1}^{(p)}-\mathbf{u}_{2}, \mu_{1}^{(p-1)}-{ }^{(p-1)} \mu_{2}^{1)}\right) \in U \times V K_{n}$ and since $\mathscr{F}$ is positive semidefinite for all admissible functions from (2.19), one obtains

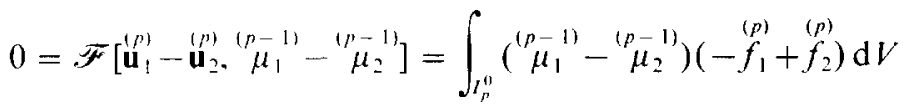

$$
\begin{aligned}
& \left.\Rightarrow \stackrel{(p-1)}{\mu_{1}} \stackrel{(p)}{f_{2}}={ }^{(p-1)} \mu_{2}\right)_{1}^{(p)}=0 \quad \forall \mathbf{x} \in I_{p}^{0}=I_{1}^{(1)},
\end{aligned}
$$

since $\stackrel{(p-1)}{\mu} \geqslant 0$ and $\stackrel{(p)}{f} \leqslant 0$ (from $(2.16) I_{p+1}^{+} \cup I_{p+1}^{0} \cup I_{p+1}^{-}=I_{p}^{0}$ ). At this stage, and without impairing the problem's generality it is additionally assumed that the principal

+ Note: The case $p>q$ can be reduced to an angular bifurcation by taking $p^{\prime}=q$ with $\lambda_{p}=0 . \ln (2.20)$ $\mathbf{u}_{\varphi}$ always $\neq 0$ but $\lambda_{p}$ may vanish. 
solution satisfies $I_{p}^{0}=I_{p+1}^{+}+$This assumption, which is equivalent to the statement that (in the principal solution) all points of the plastic zone-except perhaps the boundary points - are plastically loading, i.e. ${ }^{(p-1)} \mu>0$ almost everywhere on $I_{p}^{0}=I_{1}^{0}$, is met by the vast majority of application problems. Hence from (2.24) follows :

$$
\stackrel{(p)}{f_{i}}=0 \text { on } I_{p}^{0} \Rightarrow \stackrel{(p-1)}{\mu_{i}}=-\frac{\partial f}{\partial \mathbf{A}} \cdot \frac{\partial^{2} \phi}{\partial \boldsymbol{\alpha} \partial \boldsymbol{\varepsilon}} \cdot \boldsymbol{\varepsilon},{ }_{, k}^{(p)}\left(\mathbf{u}_{i}\right) /\left(\frac{\partial f}{\partial \mathbf{A}} \cdot \frac{\partial^{2} \phi}{\partial \boldsymbol{\alpha} \partial \boldsymbol{\alpha}} \cdot \frac{\partial f}{\partial \mathbf{A}}\right) \quad(\mathrm{i}=1,2)
$$

Supposing for simplicity that at bifurcation, the eigenmode $(\tilde{\mathbf{u}}, \bar{\mu})$ of the functional $\mathscr{F}$ in (2.9) is unique, and employing (2.20) and (2.25) (note $\mathbf{u}_{q}=\overline{\mathbf{u}}$ ), one has for the bifurcated solution, assuming at first an angular bifurcation, i.e. $p=q$,

$$
\begin{aligned}
-\left\{\lambda_{p}\left[\frac{\partial f}{\partial \mathbf{A}} \cdot \frac{\partial^{2} \phi}{\partial \boldsymbol{\alpha} \partial \boldsymbol{\varepsilon}} \cdot \boldsymbol{\varepsilon}_{u}\left(\frac{\mathrm{d} \mathbf{u}}{\mathrm{d} \lambda}\right)\right]\right. & \\
& \left.+\left[\frac{\partial f}{\partial \mathbf{A}} \cdot \frac{\partial^{2} \phi}{\partial \boldsymbol{\alpha} \partial \boldsymbol{\varepsilon}} \cdot \boldsymbol{\varepsilon}_{,_{u}}(\overline{\mathbf{u}})\right]\right\} /\left(\frac{\partial f}{\partial \mathbf{A}} \cdot \frac{\partial^{2} \phi}{\partial \boldsymbol{\alpha} \partial \boldsymbol{\alpha}} \cdot \frac{\partial f}{\partial \mathbf{A}}\right) \geqslant 0 \quad \forall \mathbf{x} \in I_{p}^{0},
\end{aligned}
$$

since (for the bifurcated solution) $\mu_{2}^{(p)} \geqslant 0$ on $I_{p}^{0}$.

Two cases are distinguished.

$$
\min _{\mathbf{x} \in I_{p}^{0}}\left[-\frac{\partial f}{\partial} \cdot \frac{\partial^{2} \phi}{\partial \mathbf{\alpha} \partial \boldsymbol{\varepsilon}} \cdot \boldsymbol{\varepsilon}_{,_{\mu}}\left(\frac{\mathrm{d} \mathbf{u}}{\mathrm{d} \lambda}\right) / \frac{\partial f}{\partial \mathbf{A}} \cdot \frac{\partial^{2} \phi}{\partial \boldsymbol{\alpha} \partial \boldsymbol{\alpha}} \cdot \frac{\partial f}{\partial \mathbf{A}}\right]>0 .
$$

This eventually occurs in structures with no unloading in their principal solution and which satisfy the loading criterion everywhere where the yield condition is satisfied. Then from (2.26)

$$
\lambda_{p} \geqslant-\frac{\partial f}{\partial \mathbf{A}} \cdot \frac{\partial^{2} \phi}{\partial \alpha \partial \boldsymbol{\varepsilon}} \cdot \boldsymbol{\varepsilon}_{, u}(\overline{\mathbf{u}}) / \frac{\partial f}{\partial \mathbf{A}} \cdot \frac{\partial^{2} \boldsymbol{\phi}}{\partial \boldsymbol{\alpha} \partial \boldsymbol{\varepsilon}} \cdot \varepsilon_{, u}\left(\frac{\mathrm{d} \mathbf{u}}{\mathrm{d} \lambda}\right)_{c}
$$

for $\mathbf{x}$ in the set $I_{p}^{0}$.

Given that the bifurcated solution exhibits no unloading in $I_{p}^{0}$, such a solution can be constructed if one introduces a priori the value

$$
\tilde{i}_{p}=\max _{\mathbf{x} \in I_{n}^{0}}\left\{-\frac{\partial f}{\partial \mathbf{A}} \cdot \frac{\partial^{2} \boldsymbol{\phi}}{\partial \alpha \partial \boldsymbol{\varepsilon}} \cdot \boldsymbol{\varepsilon}_{,_{u}}(\overline{\mathbf{u}}) / \frac{\partial f}{\partial \mathbf{A}} \cdot \frac{\partial^{2} \phi}{\partial \boldsymbol{\alpha} \partial \boldsymbol{\varepsilon}} \cdot \boldsymbol{\varepsilon}_{r_{u}}\left(\frac{\mathrm{d} \mathbf{u}}{\mathrm{d} \lambda}\right)\right\},
$$

where the maximum exists and is finite in view of (2.27).

Since at bifurcation the sign of the eigenmode can be either positive or negative, one might expect two different values for $\chi_{p}$ associated respectively with $\pm \overline{\mathbf{u}}$, one of which is necessarily positive. In most applications, due to the symmetries of the structure, both these maxima coincide and are positive. Hence of the two possible (corresponding to $\pm \overline{\mathbf{u}}$ respectively) branches at $\lambda=\lambda_{c}$, at least one will take place

${ }_{+}^{+}$Note: The equality of sets in this case is in the almost everywhere sense, i.e. the two sets differ by a set of Lebesque measure zero where $i=1$ is the principal and $i=2$ is the bifurcated solution. 
under an increasing applied load, i.e. the corresponding $\tilde{\lambda}_{p}>0$ as first discussed by SHANLEY (1947) for the column case and subsequently generalized by HILL (1958).

The bifurcation analysis proceeds in the following way: If $\lambda_{n}>\pi_{p}$, then the value of $\lambda_{p}$ is found from the higher order terms expansion of the equilibrium equation (2.6). Of course, one has to check the validity of the assumption $\lambda_{p}>\hat{i} p$. For additional details in the special case of $p=1$, the interested reader is referred to HutCHINSON (1973) (who follows Hill's approach instead of the generalized standard formalism employed here). The other possible alternative is $\lambda_{p}=\tilde{\lambda}_{p}$, which implies the existence of a point $\mathbf{x}^{*} \in I_{p}^{0}$ for which unloading starts in the bifurcated branch of the solution, this being the case for the majority of the applications treated to date.

It should also be remarked here that in most of the applications presented in the literature thus far which satisfy (2.27), their principal solutions have the additional property $I_{1}^{0}=I_{n}^{0}=I_{p+1}^{+}=\Omega$ (i.e. the principal solution satisfies everywhere in the solid the total loading condition). This property however is not necessary for (2.27) to be valid and one can easily find such applications (for example the buckling of a tapered column).

Hence, if at the critical load the principal solution satisfies (2.27), an angular bifurcation is possible (with $p=q$ ) and $\lambda_{p}$, the first term in the asymptotic expansion for the load parameter $\lambda$, has to satisfy (2.28). The value of the integer $p$, as well as the information on whether (2.28) is satisfied as a strict inequality or as an equality, depend on considerations from higher order terms.

The second case of interest is obviously:

$$
\min _{\mathbf{x} \in !_{n}^{0}}\left[-\frac{\partial f}{\partial \mathbf{A}} \cdot \frac{\partial^{2} \phi}{\partial \boldsymbol{\alpha} \partial \boldsymbol{\varepsilon}} \cdot \boldsymbol{\varepsilon}_{, \mu}\left(\frac{\mathrm{d} \mathbf{u}}{\mathrm{d} \hat{\lambda}}\right) / \frac{\partial f}{\partial \mathbf{A}} \cdot \frac{\partial^{2} \phi}{\partial \alpha \partial \boldsymbol{\alpha}} \cdot \frac{\partial f}{\partial \mathbf{A}}\right]=0,
$$

i.e. there is neutral loading in the principal solution at certain points $\mathbf{x}_{0} \in I_{p}^{0}$. In the majority of applications, these points belong to a portion $\Gamma$ of the boundary $\partial I_{p}^{0}$.

If one continues to adopt the angular bifurcation assumption that $p=q$ as in the previous case, the search for a bifurcated branch with the same loading zone $I_{p}^{0}$ leads again to inequality (2.28), which is available $\forall \mathbf{x} \in I_{p}^{0}$.

If $\mathbf{x}_{0} \in \Gamma$. then (2.30) implies that

$$
\left.\frac{\partial f}{\partial \mathbf{A}} \cdot \frac{\partial^{2} \phi}{\partial \alpha \partial \boldsymbol{\varepsilon}} \cdot \boldsymbol{\varepsilon}_{u_{u}}\left(\frac{\mathrm{d} \mathbf{u}}{\mathrm{d} \lambda}\right)\right|_{c} \mathbf{x}_{o_{0}}=0
$$

in view of the denominator's strict positivity. The inequality (2.28) leads, when $\mathbf{x} \rightarrow \mathbf{x}_{0} \in \Gamma$, necessarily to a contradiction if

$$
\left.\lim _{\mathbf{x} \rightarrow \boldsymbol{x}_{0,}, \mathbf{x} \boldsymbol{r} \mathrm{r}} \frac{\partial f}{\partial \mathbf{A}} \cdot \frac{\partial^{2} \phi}{\partial \boldsymbol{\alpha} \partial \boldsymbol{\varepsilon}} \cdot \boldsymbol{\varepsilon}_{u_{u}}(\overline{\mathbf{u}})\right|_{\mathbf{x}} \neq 0,
$$

since the right-hand side of (2.28) is unbounded in $I_{p}^{0}$.

In this condition, one concludes that no such $\lambda_{p}$ can be found: this renders the angular bifurcation impossible and hence a tangent bifurcation with $p<q$ has to be investigated. 
Note that for this case (i.e. if (2.30) is satisfied) an angular bifurcation is still possible if the eigenmode $\overline{\mathbf{u}}$ satisfies

$$
\left.\frac{\partial f}{\partial \mathbf{A}} \cdot \frac{\partial^{2} \phi}{\partial \boldsymbol{\alpha} \partial \boldsymbol{\varepsilon}} \cdot \boldsymbol{\varepsilon}_{,_{u}}(\overline{\mathbf{u}})\right|_{\mathbf{x}_{0}}=0 \quad \forall \mathbf{x}_{0} \in \Gamma
$$

and is such that

$$
\lim _{\mathbf{x} \rightarrow \mathbf{x}_{0}}\left|-\frac{\partial f}{\partial \mathbf{A}} \cdot \frac{\partial^{2} \phi}{\partial \alpha \partial \boldsymbol{\varepsilon}} \cdot \boldsymbol{\varepsilon}_{\mu_{u}}(\overline{\mathbf{u}})\right| \frac{\partial f}{\partial \mathbf{A}} \cdot \frac{\partial^{2} \phi}{\partial \alpha \partial \boldsymbol{\varepsilon}} \cdot \boldsymbol{\varepsilon}_{u_{u}}\left(\frac{\mathrm{du}}{\mathrm{d} \lambda}\right) \mid<+\infty, \quad \forall \mathbf{x}_{0} \in \Gamma .
$$

In the interest of simplicity, it will be assumed for the following general discussion that $p=1, q=2$. Thus the general methodology for the determination of the postbifurcation expansion, in the case of a tangent bifurcation, as well as the related difficulties, will be explained in a simpler context without significant loss of generality.

In view of the assumption $p=1, q=2$ the solution to the rate 1 problem (see (2.8)) is unique, but the solution to the rate 2 problem (see (2.11)) is not unique at $\lambda=\lambda_{\text {c }}$. The explicit form of the rate 2 equilibrium equation, i.e. the $O\left(\tau^{2}\right)$ term in the expansion of the equilibrium Eq. (2.6) is

$$
\begin{aligned}
& \int_{\Omega}\left\{\boldsymbol { \varepsilon } _ { u } ( \delta \mathbf { u } ) \cdot \left[\frac{\partial^{2} \phi}{\partial \varepsilon \partial \boldsymbol{\varepsilon}} \cdot \tilde{\varepsilon}+\frac{\partial^{2} \phi}{\partial \varepsilon \partial \alpha} \cdot \frac{\partial f}{\partial \mathbf{A}} \ddot{\mu}+\left(\frac{\partial^{3} \phi}{\partial \boldsymbol{\varepsilon} \partial \boldsymbol{\varepsilon} \partial \boldsymbol{\varepsilon}} \cdot \dot{\varepsilon}+\frac{\partial^{3} \phi}{\partial \boldsymbol{\varepsilon} \partial \boldsymbol{\varepsilon} \partial \boldsymbol{\alpha}} \cdot \frac{\partial f}{\partial \mathbf{A}} \mu\right) \cdot \dot{\boldsymbol{\varepsilon}}\right.\right. \\
& \left.+\left(\frac{\partial^{3} \phi}{\partial \boldsymbol{\varepsilon} \partial \boldsymbol{\alpha} \dot{\partial \varepsilon}} \cdot \dot{\varepsilon}+\frac{\partial^{3} \phi}{\partial \varepsilon \partial \boldsymbol{\alpha} \partial \boldsymbol{\alpha}} \cdot \frac{\partial f}{\partial \mathbf{A}} \mu\right) \cdot \frac{\partial f}{\partial \mathbf{A}} \mu-\frac{\partial^{2} \phi}{\partial \varepsilon \partial \boldsymbol{\alpha}} \cdot \frac{\partial^{2} f}{\partial \mathbf{A} \partial \mathbf{A}} \cdot\left(\frac{\partial^{2} \phi}{\partial \boldsymbol{\alpha} \partial \boldsymbol{\varepsilon}} \cdot \dot{\boldsymbol{\varepsilon}}+\frac{\partial^{2} \phi}{\partial \boldsymbol{\alpha} \partial \boldsymbol{\alpha}} \cdot \frac{\partial f}{\partial \mathbf{A}} \mu\right) \mu\right] \\
& \left.+2 \boldsymbol{\varepsilon}_{, u u}(\dot{\mathbf{u}}, \delta \mathbf{u}) \cdot\left[\frac{\partial^{2} \phi}{\partial \boldsymbol{\varepsilon} \partial \boldsymbol{\varepsilon}} \cdot \dot{\boldsymbol{\varepsilon}}+\frac{\partial^{2} \phi}{\partial \boldsymbol{\varepsilon} \partial \boldsymbol{\alpha}} \cdot \frac{\partial f}{\partial \mathbf{A}} \mu\right]+\frac{\partial \phi}{\partial \varepsilon} \cdot\left[\boldsymbol{\varepsilon}_{\varphi_{u u u}}(\dot{\mathbf{u}}, \dot{\mathbf{u}}, \delta \mathbf{u})+\boldsymbol{\varepsilon}_{, u u}(\ddot{\mathbf{u}}, \delta \mathbf{u})\right]\right\} \mathrm{d} V \\
& +\int_{\partial I_{2}^{-}} \boldsymbol{\varepsilon}_{\mu}(\delta \mathbf{u}) \cdot \frac{\partial^{2} \phi}{\partial \boldsymbol{\varepsilon} \partial \boldsymbol{\alpha}} \cdot \frac{\partial f}{\partial \mathbf{A}} \llbracket \mu \rrbracket v_{1} \mathrm{~d} S-\ddot{\lambda} \int_{\boldsymbol{\Omega} \Omega} \mathbf{T}^{0} \cdot \delta \mathbf{u}
\end{aligned}
$$

where

$$
\begin{gathered}
\mu=-\chi_{l^{\ddagger}}\left(\frac{\partial f}{\partial \mathbf{A}} \cdot \frac{\partial^{2} \phi}{\partial \boldsymbol{\alpha} \partial \boldsymbol{\varepsilon}} \cdot \dot{\varepsilon} / \frac{\partial f}{\partial \mathbf{A}} \cdot \frac{\partial^{2} \phi}{\partial \boldsymbol{\alpha} \partial \boldsymbol{\alpha}} \cdot \frac{\partial f}{\partial \mathbf{A}}\right), \quad \dot{\boldsymbol{\varepsilon}}=\boldsymbol{\varepsilon},{ }_{u}(\dot{u}), \\
\llbracket \boldsymbol{\alpha} \nabla \rrbracket \cdot \mathbf{n} v_{1}+\llbracket \dot{\boldsymbol{\alpha}} \rrbracket=0 \quad \text { with } \llbracket \dot{\boldsymbol{\alpha}} \rrbracket=\llbracket \mu \rrbracket \frac{\partial f}{\partial \mathbf{A}},
\end{gathered}
$$

with analogous expressions for $\dot{\mu}$ and $\ddot{\varepsilon}$. In addition $\llbracket \mu \rrbracket$ denotes the jump in $\mu$ and $v_{1}$ denotes the velocity of the boundary $\partial I_{2}^{+}$of $I_{2}^{+}$in the direction of its outward normal $\mathbf{n}$. Note that in the derivation of the rate 2 equilibrium equation, account is taken of the fact that in general $\mu(x)$ is a discontinuous function on the surface $\partial I_{2}^{+}$(recall from (2.11) that $\mu>0$ on $I_{2}^{+}$and $\mu=0$ on $\left.\Omega \backslash I_{2}^{+}\right)$. 
In the interest of computational simplification and without impairing significantly the problem's generality, it is further assumed that the strain rates $\dot{\varepsilon}, \ddot{\varepsilon}, \ldots$ are continuous functions of $\mathbf{x}$. In contrast the rates of the internal variable $\dot{\alpha}, \ddot{\alpha}, \ldots$ having moving discontinuities which are heing taken into account.

Applying successively (2.35) to the principal and the bifurcated solutions respectively one obtains as expected (recalling that $i_{i}=\lambda_{c}+\lambda_{1} \tau+\cdots \cdot \mathbf{u}=\mathbf{u}_{0}(\lambda)+$ $\left.\mathbf{u}_{2}\left(\tau^{2} / 2\right)+\mathbf{u}_{3}\left(\tau^{3} / 6\right)+\cdots\right)$

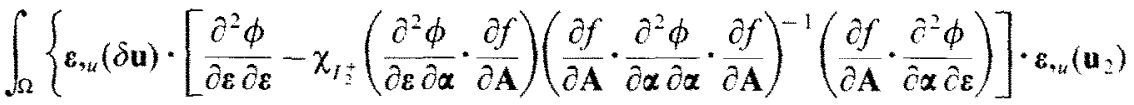

$$
\begin{aligned}
& \left.+\frac{\partial \phi}{\partial \boldsymbol{\varepsilon}} \cdot \varepsilon_{\mathrm{su}_{u}}\left(\mathbf{u}_{2}, \delta \mathbf{u}\right)\right\} \mathrm{d} V=0 .
\end{aligned}
$$

with the functional evaluated on the principal branch at $\lambda=\lambda_{c}$.

The determination of $\lambda_{1}$ will require the $\mathrm{O}\left(\tau^{3}\right)$ term in the expansion of the equilibrium equation (2.6). By taking rates in (2.35) and applying the result successively to the principal and bifurcated branch of the solution one obtains after some lengthy but straightforward calculations (in which 2.36) is also taken into account)

$$
\begin{gathered}
3 \hat{\lambda}_{1} \int_{\Omega} \frac{\mathrm{d}}{\mathrm{d} \lambda}\left\{\boldsymbol{\varepsilon}_{y_{u}}\left(\mathbf{u}_{2}\right) \cdot\left[\frac{\partial^{2} \phi}{\partial \boldsymbol{\varepsilon} \partial \boldsymbol{\varepsilon}}-\chi_{I_{2}^{+}}\left(\frac{\partial^{2} \phi}{\partial \boldsymbol{\varepsilon} \partial \boldsymbol{\alpha}} \cdot \frac{\partial f}{\partial \mathbf{A}}\right)\left(\frac{\partial f}{\partial \mathbf{A}} \cdot \frac{\partial^{2} \phi}{\partial \boldsymbol{\alpha} \boldsymbol{\partial \alpha}} \cdot \frac{\partial f}{\partial \mathbf{A}}\right)^{-1}\left(\frac{\partial f}{\partial \mathbf{A}} \cdot \frac{\partial^{2} \phi}{\partial \alpha \partial \boldsymbol{\varepsilon}}\right)\right] \cdot \boldsymbol{\varepsilon}_{,_{u}}\left(\mathbf{u}_{2}\right)\right. \\
\left.+\frac{\partial \phi}{\partial \boldsymbol{\varepsilon}} \cdot \boldsymbol{\varepsilon}_{,_{u u}}\left(\mathbf{u}_{2}, \mathbf{u}_{2}\right)\right\} \mathrm{d} V+\Delta\left[\int_{e I_{2}^{+}} \boldsymbol{\varepsilon}_{,_{u}}\left(\mathbf{u}_{2}\right) \cdot \frac{\partial^{2} \phi}{\partial \boldsymbol{\varepsilon} \partial \boldsymbol{\alpha}} \llbracket \mu \rrbracket v_{1} \mathrm{~d} S\right] \\
+\Delta\left[\int_{i I_{2}^{+}} \boldsymbol{\varepsilon}_{w u}\left(\mathbf{u}_{2}\right) \cdot \frac{\partial^{2} \phi}{\partial \boldsymbol{\varepsilon} \partial \boldsymbol{\alpha}} \llbracket \dot{\mu} \rrbracket v_{2} \mathrm{~d} S\right]=0
\end{gathered}
$$

where $\Delta f \equiv \lim _{\tau \rightarrow 0}\left(f_{2}(\tau)-f_{1}(\tau)\right)$ where subscripts 1 and 2 denote quantities evaluated on the principal and bifurcated branch respectively. Obviously the surface integrals also depend on $\lambda_{1}$ but in a highly complicated fashion which requires the details of motion near $\lambda=\lambda_{c}$ of the principal and bifurcated surfaces $\partial I_{2}^{+}$which separate the plastically loading zones from the corresponding elastic ones.

An application of the analysis to the bifurcation of an elastic-plastic structure will be presented in the next section, where the computational complications due to the propagating discontinuities become apparent.

It should also be mentioned here, that for the special case of an angular bifurcation (in which also $I_{p}^{0}=\Omega$ ) a comprehensive postbifurcation analysis based on the propagation of the unloading zone was first presented by HuTchiNSON (1973) using Hill's plasticity formulation and requiring fractional expansions. More recently some of the problems treated by HuTCHINSON (1974), were repeated by NGUYEN (1987) using the standard generalized material formulation and an integral series expansion of the type proposed in (2.20). All the aforementioned work concerned angular bifurcations with $I_{p}^{0}=\Omega$, a fact which simplifies the analysis considerably. 


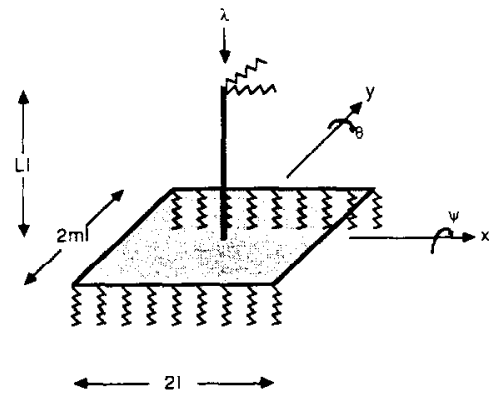

FIG. 1. A model which exhibits both an angular and a smooth bifurcation.

While a first attempt to address the problem of smooth bifurcation was made by TrIaNTAFYlLIDIS (1983) using a different line of attack (i.e. considering a set of rate invariant constitutive laws proposed by CHRISTOFFERSEN and HuTCHINSON, 1979) the present methodology provides a unified approach for the study of elastic-plastic bifurcation problems without any of the restrictive assumptions related to the principal solution that have been adopted in the literature thus far.

\section{Illustrative Example}

An example is considered which is capable of exhibiting both an angular as well as a smooth bifurcation. This example, which is in the spirit of the finite degree of freedom models used by Shanley (1947) (see also Hutchinson, 1974; NGuYen, 1987) has been employed by TRIANTAFYLLIDIS (1983) to show the possibility of a smooth bifurcation in an elastic-plastic solid.

The model consists of a horizontal rigid rectangular plate of dimensions $2 l \times 2 \mathrm{ml}$ resting on two lines of continuously distributed elastic-plastic springs as shown in Fig. 1. A straight rod of length $L l$ is rigidly attached perpendicularly to the plate's center on one end while the other end is attached to two coupled nonlinear springs (i.e. such that the displacement in one of them induces a force in the other) one in the $x^{\prime}$ and the other in the $y^{\prime}$ direction as shown in Fig. 1.

The externally applied vertical load is $\lambda E l^{2}$ where $\lambda$ is the load parameter of the problem and $E$ is the modulus in the elastic region for the bilinear hardening elasticplastic springs. The structure has three degrees of freedom, namely the two rotations $\theta$ and $\psi$ about the $+y^{\prime}$ and $-x^{\prime}$ axes respectively and the vertical displacement $u l$ (note that $\lambda, u, \theta, \psi$ are dimensionless). Under the action of the vertical load $\lambda$, the structure which initially follows a path $u \neq 0, \theta=0, \psi=0$ bifurcates first at $\lambda=\lambda_{\theta}$ to a solution with $u \neq 0, \theta \neq 0, \psi=0$ and upon a further increase of $\lambda$ it bifurcates again at $\lambda=\lambda_{\psi}>\lambda_{\theta}$ (it is tacitly assumed that $m^{2}>1 / 3$ ) to a solution with $u \neq 0$, $\theta \neq 0, \psi \neq 0$. It will be shown that the first bifurcation is an angular one while the second one is a smooth bifurcation.

The total energy stored in the system is 


$$
\begin{aligned}
& \mathscr{E}=\frac{1}{2} \int_{-l}^{l}\left[\phi\left(x^{\prime}, m l\right)+\phi\left(x^{\prime},-m l\right)\right] \mathrm{d} x^{\prime}-\lambda E l^{3}\left[u+\frac{L}{2}\left(\theta^{2}+\psi^{2}\right)\right]-E l^{3} K L \theta^{\frac{\psi^{2}}{2}}, \\
& \phi\left(x^{\prime}, y^{\prime}\right)=\frac{1}{2} E\left\{\left[\varepsilon^{\prime}\left(x^{\prime}, y^{\prime}\right)-x^{\prime}\left(x^{\prime}, y^{\prime}\right)\right]^{2}+h\left[\alpha^{\prime}\left(x^{\prime}, y^{\prime}\right)\right]^{2}\right\}, \\
& \frac{\varepsilon^{\prime}}{l} \equiv \varepsilon\left(x^{\prime}, y^{\prime}\right)=u+\frac{x^{\prime}}{l} \theta+\frac{y^{\prime}}{l} \psi, \quad-1 \leqslant \frac{x^{\prime}}{l} \equiv x \leqslant 1, \quad \frac{y^{\prime}}{l} \equiv y= \pm m, \\
& \frac{\alpha^{\prime}}{l} \equiv x, \quad A^{\prime}=-\frac{\partial \phi}{\partial \alpha^{\prime}}=E l[(1+h) \alpha-\varepsilon]=l E A, \\
& A^{\prime} \in C^{\prime} \equiv[-k E l, k E l] \Leftrightarrow A \in C \equiv[-k, k],
\end{aligned}
$$

where primed quantities are dimensional ones while unprimed quantities designate their dimensionless counterparts.

The internal variable $\alpha^{\prime}$ in this case represents the plastic strain in the kinematically hardening bilinear model, while the associated force $A^{\prime}$ is the corresponding backstress which is bounded by the yield stress $k^{\prime}=E l k$, with $k$ the dimensionless yield stress. It is also noted that the third term in the expression for the energy of the system is due to the coupled nonlinear springs at the end of the vertical rod.

The constitutive and equilibriums Eqs (2.1), (2.3) and (2.6) employed in (3.1) yield

$$
\begin{aligned}
\lambda & =4 u-\int_{-1}^{1}[\alpha(x, m)+\alpha(x,-m)] \mathrm{d} x, \\
\lambda L \theta & =-K L \frac{\psi^{2}}{2}+\frac{4}{3} \theta-\int_{-1}^{1}[\alpha(x, m)+\alpha(x,-m)] x \mathrm{~d} x . \\
\lambda L \psi & =-K L \psi \theta+4 m^{2} \psi-\int_{-1}^{1}[\alpha(x, m)-\alpha(x,-m)] m \mathrm{~d} x, \\
\dot{\alpha} & =0 \text { if }|(1+h) \alpha-\varepsilon|<k, \quad \dot{\alpha} \geqslant 0 \text { if }|(1+h) \alpha-\varepsilon|=k .
\end{aligned}
$$

In view of the relative analytic simplicity of the problem an exact solution for the primary $(\theta)$ bifurcation (branch $u \neq 0, \theta \neq 0, \psi=0$ ) is available and a complete asymptotic solution for the secondary $(\psi)$ bifurcation (branch $u \neq 0, \theta \neq 0, \psi \neq 0$ ) can be constructed. The specialization of the general results presented in Section 2.2 to the present example will also be discussed.

\subsection{Primary $(\theta)$ bifurcation (angular case, $\mathrm{p}=\mathrm{q}=2$ )}

The primary $(\theta)$ bifurcation of the plate is identical to the rigid $T$ bifurcation example presented repeatedly in the literature (see for example HuTCHINSON, 1974; NGUYEN, 1987). However, only the asymptotic behavior near the critical load $\lambda_{\theta}$ was of interest and the full solution for the primary $(\theta)$ branch, which is necessary for calculations in the secondary $(\psi)$ bifurcation has not been presented in the literature thus far. For this reason, as well as for the completeness of the presentation the primary $(\theta)$ bifurcation of the studied example will be given here. 
Starting with the rate form of (3.2) and considering the tilted equilibrium position with $u \neq 0, \theta \neq 0, \psi=0$ for which the length of the unloading zone is $\tau$, i.e. $\dot{\varepsilon}=0$ at $x_{n}=\tau-1$ where $x_{n}$ is the coordinate of the neutral loading zone, one deduces (assuming for simplicity $\theta$ to be the time-like parameter for this particular derivation, i.e. ()$\left.^{\cdot} \equiv \mathrm{d}() / \mathrm{d} \theta\right)$,

$$
\begin{aligned}
\lambda & =-\frac{1}{1+h}\left(\dot{u}^{2}+1\right)+2 \frac{1+2 h}{1+h} \dot{u}, \\
L(\lambda \theta) & =\frac{1}{3} \frac{1}{1+h} \dot{u}^{3}-\frac{1}{1+h} \dot{u}+\frac{2}{3} \frac{1+2 h}{1+h}, \\
\text { at } \theta & =0, \quad u=u_{\theta}=\frac{1}{3 L}-\frac{k}{h}, \quad \lambda=\lambda_{\theta}=\frac{4}{3 L} \frac{h}{1+h},
\end{aligned}
$$

assuming, of course, $L k>h / 3(1+h)$ in order for the $(\theta)$ bifurcation to occur in the plastic range. The above system can be integrated by considering $\theta$ as the dependent variable and $\tau^{\prime} \equiv 1-\dot{u}$ as the independent one to give

$$
\begin{aligned}
\theta(\tau) & =\frac{1}{2 L}\left[4 h(1-\tau)-\tau^{2}\right]^{-1 / 2} \int_{0}^{\tau} \tau^{\prime}\left(2-\tau^{\prime}\right)\left[4 h\left(1-\tau^{\prime}\right)-\tau^{\prime 2}\right]^{-1 / 2} \mathrm{~d} \tau^{\prime}, \\
u(\tau) & =\frac{1}{3 L}-\frac{k}{h}+\int_{0}^{\tau}\left(1-\tau^{\prime}\right) \frac{\mathrm{d} \theta}{\mathrm{d} \tau^{\prime}} \mathrm{d} \tau^{\prime}, \\
\lambda(\tau) & =\frac{4}{3 L} \frac{h}{1+h}+\int_{0}^{\tau}\left[\frac{4 h\left(1-\tau^{\prime}\right)-\tau^{\prime 2}}{1+h}\right] \frac{\mathrm{d} \theta}{\mathrm{d} \tau^{\prime}} \mathrm{d} \tau^{\prime}, \\
\alpha(x, m) & =\alpha(x,-m) \\
& = \begin{cases}{[u(1+x)+x \theta(1+x)-k] /(1+h)} & \text { for } \tau-1 \geqslant x \text { (unloading zone) } \\
{[u(\tau)+x 0(\tau)-k] /(1+h)} & \text { for } \tau-1 \leqslant x \text { (loading zone). }\end{cases}
\end{aligned}
$$

The above equations constitute the full solution for the primary $(\theta)$ bifurcation of the model which occurs at the critical load $\lambda_{\theta}=4 h / 3 L(1+h)$.

Given that the size of the unloading zone $\tau$ increases with increasing $\lambda$ (at least near $\left.\lambda=\lambda_{\theta}\right)$ and that $\tau=0$ at $\lambda=\dot{\lambda}_{\theta}$, one can consider $\tau$ as a new time-like parameter. Expanding (3.4) about $\tau=0$ yields

$$
\begin{aligned}
& \theta=0+\frac{1}{8 L h} \tau^{2}+O\left(\tau^{3}\right), \\
& u=u_{f}+\frac{1}{8 L h} \tau^{2}+O\left(\tau^{3}\right), \\
& \lambda=\hat{\lambda}_{\theta}+\frac{1}{2 L(1+h)} \tau^{2}+O\left(\tau^{3}\right) .
\end{aligned}
$$

This reconfirms that the primary $(\theta)$ bifurcation of this example (which coincides 
with the bifurcation of the literature's popular rigid $T$ model) is an angular one with $p=q=2$ (see (2.20)). A different derivation of (3.5) following the general asymptotic method in terms of integral powers of $\tau$ proposed in Section 2.2 has already been given in NGIJYEN (1987) and hence the higher order terms in (3.5) will not be of concern here. Of course, according to the general analysis presented in Section 2, a sufficient condition for angular bifurcation (provided that a bifurcated solution exists) is given by (2.27). Indeed, using (3.1) and (3.2) in (2.27) one obtains (note also that $I_{1}^{0}=$ $I_{2}^{+}=\Omega$ in the principal solution):

$$
\min _{\mathbf{x} \in \Omega}\left[-\frac{\partial f}{\partial \mathbf{A}} \cdot \frac{\partial^{2} \phi}{\partial \boldsymbol{\alpha} \partial \boldsymbol{\varepsilon}} \cdot \boldsymbol{\varepsilon}_{u}\left(\frac{\mathrm{d} \mathbf{u}}{\mathrm{d} \lambda}\right) / \frac{\partial f}{\partial \mathbf{A}} \cdot \frac{\partial^{2} \phi}{\partial \boldsymbol{\alpha} \partial \boldsymbol{\alpha}} \cdot \frac{\partial f}{\partial \mathbf{A}}\right]=\min _{-1 \leqslant x \leqslant 1}\left[\frac{(\mathrm{d} u / \mathrm{d} \lambda)_{c}}{1+h}\right]=\frac{1}{4 h}>0 .
$$

as expected from (3.5). One can also easily verify in this case that $\lambda_{2}=\tilde{\lambda}_{2}$ in view of the unloading starting at $x=-1$ at the onset of the bifurcation.

Indeed, recalling that the eigenmode $\overline{\mathbf{u}}=\mathbf{u}_{2}=\left(u_{2}, \theta_{2}, \psi_{2}\right)=\left(0,(8 L h)^{-1}, 0\right)$, from (2.29),

$$
\begin{array}{r}
\tilde{i}_{2}=\max _{\mathbf{x} \in \Omega}\left\{-\frac{\partial f}{\partial \mathbf{A}} \cdot \frac{\partial^{2} \phi}{\partial \boldsymbol{\alpha} \partial \boldsymbol{\varepsilon}} \cdot \boldsymbol{\varepsilon}_{{ }_{u}}(\mathbf{\mathbf { u }}) / \frac{\partial f}{\partial \mathbf{A}} \cdot \frac{\partial^{2} \phi}{\partial \boldsymbol{\alpha} \partial \boldsymbol{\varepsilon}} \cdot \boldsymbol{\varepsilon}_{,_{u}}\left(\frac{\mathrm{d} \mathbf{u}}{\mathrm{d} \lambda}\right)\right\}=\max _{l \leq x \leq 1}\left[\frac{-x / 8 L h}{(1+h) / 4 h}\right] \\
=\frac{1}{2 L(1+h)}=\lambda_{2}
\end{array}
$$

with the maximum occurring at $x=-1$, where unloading starts at bifurcation. exactly as expected by the general theory since $\lambda_{2}=\tilde{\lambda}_{2}$. In the derivation of (3.7) one of the two modes was considered (the one with $\theta_{2}>0$ ). Had the other eigenmode been used, the value of $\tilde{\lambda}_{2}$ would have remained the same (obviously in view of the structure's symmetry), but unloading would have started at $x=+1$.

\subsection{Secondary $(\psi)$ bifurcation (smooth case, $\mathrm{p}=1, \mathrm{q}=2$ )}

Next attention is focussed on the secondary $(\psi)$ bifurcation of the model. The results have already been presented by TRIANTAFYLLIDIS (1983) using a different approach but the derivations will be repeated here using the standard generalized material formulation. The results will also be compared with those deduced from the general analysis in Section 2.2.

Without loss of generality (in view of the structure's symmetry) it will be assumed that $\psi \geqslant 0$, in which case from (3.1) follows

$$
\begin{aligned}
& y=-m\left\{\begin{array}{llc}
\dot{\alpha}=0 & \text { for } & -1 \leqslant x \leqslant x_{-} \\
\dot{\alpha}=\frac{\dot{u}+x \dot{\theta}-m \psi}{1+h}>0 & \text { for } & x_{-}<x \leqslant 1,
\end{array}\right. \\
& y=m \quad\left\{\begin{array}{llc}
\dot{\alpha}=0 & \text { for } & 1 \leqslant x \leqslant x_{+}^{r} \\
\dot{\alpha}=\frac{\dot{u}+x \dot{\theta}+m \psi}{1+h}>0 & \text { for } & x_{+}^{r}<x \leqslant 1,
\end{array}\right.
\end{aligned}
$$

with 


$$
x_{-}=(-\dot{u}+m \dot{\psi}) / \dot{\theta}, \quad x_{+}=(-\dot{u}-m \dot{\psi}) / \dot{\theta}
$$

and $x_{+}^{r}(t)$ the root of the equation

$$
\left[u(t)-u_{0}\left(1+x_{+}^{r}\right)\right]+x_{+}^{r}\left[\theta(t)-\theta_{0}\left(1+x_{+}^{r}\right)\right]+m \psi(t)=0,
$$

where $x_{-}(t)$ and $x_{+}^{r}(t)$ are the coordinates of the boundaries separating the elastic from the plastic zone at $y=-m$ and $y=+m$ respectively, while $x_{-}$and $x_{+}$are the corresponding coordinates of the points where the strain rate $\dot{\varepsilon}=0$. The principal solution for the secondary $(\psi)$ bifurcation is obviously given by $(3.4)$ and to avoid confusion is characterized by a zcro subscript in (3.8) and subsequently. To further avoid confusion, since the time-like parameter in the angular bifurcation case was designated by $\tau$, for this case the time-like parameter is denoted by $t$ and hence ()$^{\circ} \equiv \mathrm{d}() / \mathrm{d} t$ in (3.8) and on.

Combining (3.8) with the rate form of the equilibrium equations (3.2), one obtains the following expressions governing the secondary $(\psi)$ bifurcation of the model:

$$
\begin{aligned}
& \dot{\lambda} \dot{\theta}=4 \dot{u} \dot{\theta}+\frac{1}{1+h}\left[\frac{\dot{\theta}^{2}}{2}\left(x_{+}^{r}-x_{+}\right)^{2}-(\dot{u}+\dot{\theta})^{2}-(m \dot{\psi})^{2}\right] \\
& {\left[(\lambda L \theta)^{\circ}+K L \psi \psi\right] \dot{\theta}^{2}=\frac{2}{3} \frac{1+2 h}{1+h} \dot{\theta}^{3}+\frac{1}{1+h}\left[\frac{\dot{u}^{3}}{3}\right.}+\dot{u}\left((m \psi)^{2}-\dot{\theta}^{2}\right) \\
&\left.+\frac{\dot{\theta}^{3}}{6}\left(x_{+}^{r}-x_{+}\right)^{2}\left(2 x_{+}^{r}+x_{+}\right)\right] \\
& {[(\lambda+K \theta) L \psi] \dot{\theta}=4 m^{2} \dot{\psi} \dot{\theta}+\frac{m}{1+h}\left[\frac{\dot{\theta}^{2}}{2}\left(x_{+}^{r}-x_{+}\right)^{2}-2 m \dot{\psi}(\dot{u}+\dot{\theta})\right] . }
\end{aligned}
$$

As explained in Section 3.1, the length of the elastic unloading zone $\tau$ (see Eq. (3.4)) can be adopted as the time-like parameter in the principal solution (in reality $\lambda$ is controlled but in the vicinity of the critical load $\lambda=\lambda_{\psi}, \mathrm{d} \lambda / \mathrm{d} \tau>0$, and hence $\tau$ can be chosen to play the role of the time-like parameter for the principal solution).

Using the general results obtained in Section 2, it is not difficult to see that an angular bifurcation in $(\psi)$ is impossible in this case. Indeed, noticing from (3.4) that the $(\psi)$ bifurcation eigenmode is $\overline{\mathbf{u}}=(0,0,1)$ and that from the principle solution in (3.4) $\Gamma=\{(-1+\tau, m),(-1+\tau,-m)\}$ (the set is made up of the two neutral loading points separating the loading from the unloading zones), one can easily see that both (2.31) and (2.32) are satisfied and hence no angular bifurcation in $(\psi)$ is possible. Therefore, a tangent bifurcation with $p=1, q=2$ will be adopted, with the following asymptotic expansions in $\tau(0<t \ll 1)$

$$
\begin{aligned}
\tau(t) & =\tau_{c}+\tau_{1} t+\tau_{2} \frac{t^{2}}{2}+\cdots, \\
u(t) & =u_{0}(\tau(t))+u_{2} \frac{t^{2}}{2}+u_{3} \frac{t^{3}}{6}+\cdots,
\end{aligned}
$$




$$
\begin{aligned}
& \theta(t)=\theta_{0}(\tau(t))+\theta_{2} \frac{t^{2}}{2}+\theta_{3} \frac{t^{3}}{6}+\cdots, \\
& \psi(t)=0+\psi_{2} \frac{t^{2}}{2}+\psi_{3} \frac{t^{3}}{6}+\cdots, \\
& \lambda(t)=\lambda_{0}(\tau(t)),
\end{aligned}
$$

where $\left(u_{2}, \theta_{2}, \psi_{2}\right)=(0,0,1)=\mathbf{u}_{2}$, is the structure's normalized eigenmode (see also (3.7)). Hence (3.8) is the specialization of $(2.20)$ to the finite degree of freedom example treated here with $p=1$ and $q=2$.

Employing (3.4), (3.8), and (3.10) into the rate 1 equilibrium Eq. (3.9) one obtains from the $O(t)$ term

$$
\left[\lambda_{0}\left(\tau_{c}\right)+K \theta_{0}\left(\tau_{c}\right)\right] L=2 m^{2}\left(\frac{2 h+\tau_{c}}{1+h}\right)
$$

which is the equation providing the critical load $\lambda_{\psi}=\lambda_{0}\left(\tau_{c}\right)$ (or equivalently $\tau_{c}$ ) for the secondary $(\psi)$ bifurcation of the model.

For the higher order terms in the asymptotic expansion of the equilibrium equations, the following expressions for the boundary coordinates of the plastic zones at $y= \pm m$ will be needed (see (3.8), (3.10))

$$
\begin{aligned}
& x_{+}^{r}(t)=[\tau(t)-1]+x_{1}^{r} t+x_{2}^{r} \frac{t^{2}}{2}+\cdots ; \quad x_{1}^{r}=-\left(m / \frac{\mathrm{d} \theta_{0}}{\mathrm{~d} \tau}\right)^{1: 2} . \\
& x_{+}(t)=[\tau(t)-1]+x_{1}^{+} t+x_{2}^{+} \frac{t^{2}}{2}+\cdots ; \quad x_{1}^{+}=-m / \tau_{1} \frac{\mathrm{d} \theta_{0}}{\mathrm{~d} \tau} . \\
& x_{-}(t)=[\tau(t)-1]+x_{1} t+x_{2} \frac{t^{2}}{2}+\cdots ; \quad x_{1}^{-}=m / \tau_{1} \frac{\mathrm{d} \theta_{0}}{\mathrm{~d} \tau} .
\end{aligned}
$$

Note that at the principal branch $\left(x_{+}^{r}\right)^{0}=x_{+}^{0}=x_{-}^{0}=\tau-1$.

Continuing with the $\mathrm{O}\left(t^{2}\right)$ term in the rate equilibrium equation (3.9) one obtains (by using also (3.4), (3.8), (3.10)-(3.12))

$$
\left[3\left(\frac{\mathrm{d} \lambda_{0}}{\mathrm{~d} \tau}+K \frac{\mathrm{d} \theta_{0}}{\mathrm{~d} \tau}\right) L-4 \frac{m^{2}}{1+h}\right] \frac{\mathrm{d} \theta_{0}}{\mathrm{~d} \tau}\left(\tau_{1}\right)^{2}=\frac{m}{1+h}\left[m+\frac{\mathrm{d} \theta_{0}}{\mathrm{~d} \tau} \tau_{1} x_{1}^{r}\right]^{2} .
$$

which finally leads to the following expression for $\tau_{\text {! }}$

$$
\tau_{1}=\left(m / \frac{\mathrm{d} \theta_{0}}{\mathrm{~d} \tau}\right)^{1 / 2}\left\{1 \pm\left[3\left(\frac{1+h}{m^{2}}\right)\left(\frac{\mathrm{d} \hat{\lambda}_{0}}{\mathrm{~d} \tau}+K \frac{\mathrm{d} \theta_{0}}{\mathrm{~d} \tau}\right) L-4\right]^{1 / 2}\right\}^{-1},
$$

where it is understood that all derivatives of the principal solution are evaluated at $\tau=\tau_{\text {. }}$.

At this point it should also be noted that for adequately large $K,(3.11)$ will admit a solution (with $\tau_{r} \rightarrow 0$ as $K \rightarrow \infty$ ) and the expression for $\tau_{1}$ (3.14) will also make sense. Only solutions with $\tau_{1}>0$ are acceptable (in view of the initial assumption that 
the controlled parameter in the experiment is the external load $\lambda$ which is considered to increase monotonically). It appears from (3.14) that for certain values of $\tau_{c}$, instead of one, two different secondary $(\psi)$ bifurcation branches are possible for $\psi>0$ (and also another two are possiblc for $\psi<0$ ).

The same results for $\tau_{1}$ could have been obtained by a direct application of (2.37) to the present model. Noting that

$$
\begin{aligned}
& 3 \lambda_{1} \frac{\mathrm{d}}{\mathrm{d} \lambda} \int_{\Omega}\left\{\boldsymbol{\varepsilon}_{\mu_{u}}\left(\mathbf{u}_{2}\right) \cdot\left[\frac{\partial^{2} \phi}{\partial \boldsymbol{\varepsilon} \partial \boldsymbol{\varepsilon}}-\chi_{I^{+}}\left(\frac{\partial^{2} \phi}{\partial \boldsymbol{\varepsilon} \partial \boldsymbol{\alpha}} \cdot \frac{\partial f}{\partial \mathbf{A}}\right)\left(\frac{\partial f}{\partial \mathbf{A}} \cdot \frac{\partial^{2} \phi}{\partial \boldsymbol{\alpha} \partial \boldsymbol{\alpha}} \cdot \frac{\partial f}{\partial \mathbf{A}}\right)^{-1}\left(\frac{\partial f}{\partial \mathbf{A}} \cdot \frac{\partial^{2} \phi}{\partial \boldsymbol{\alpha} \partial \boldsymbol{\varepsilon}}\right)\right] \cdot \boldsymbol{\varepsilon}_{,_{u}}\left(\mathbf{u}_{2}\right)\right. \\
& \left.+\frac{\partial \phi}{\partial \boldsymbol{\varepsilon}} \cdot \boldsymbol{\varepsilon}_{,_{u и}}\left(\mathbf{u}_{2}, \mathbf{u}_{2}\right)\right\} \mathrm{d} V=3 \tau_{1}\left(\frac{\mathrm{d} \dot{\lambda}_{0}}{\mathrm{~d} \tau}+K \frac{\mathrm{d} \theta_{0}}{\mathrm{~d} \tau}\right)_{\tau=\tau_{c}}, \\
& \Delta\left[\int_{\partial r_{2}^{+}} \boldsymbol{\varepsilon}_{{ }_{u}}\left(\mathbf{u}_{2}\right) \cdot \frac{\partial^{2} \phi}{\partial \boldsymbol{\varepsilon} \partial \boldsymbol{\alpha}} \llbracket \mu \rrbracket v_{1} \mathrm{~d} S\right]=\left[-\frac{m}{1+h}\left(\dot{u}+x_{+}^{r} \dot{\theta}+m \psi\right) \dot{x}_{+}^{r}\right]_{x=\tau_{c}-1 . t=0} . \\
& \Delta\left[\int_{i I_{2}^{+}} \boldsymbol{\varepsilon}_{,_{u}}\left(\mathbf{u}_{2}\right) \cdot \frac{\partial^{2} \phi}{\partial \boldsymbol{\varepsilon} \partial \alpha} \llbracket \dot{\mu} \rrbracket v_{2} \mathrm{~d} S\right]=\left\{-\frac{m}{1+h}\left[-(\ddot{u}+x \ddot{\theta}-m \psi) \dot{x}_{-}\right.\right. \\
& \left.\left.+(\ddot{u}+x \ddot{\theta}+m \psi) \dot{x}_{+}^{r}\right]\right\}_{x=\tau_{c}-1, t=0},
\end{aligned}
$$

one recovers (3.14). It should be noted however that the relation between $x_{1}^{r}$ and the principal solution depends on the details of the propagating discontinuity of $\mu$ along $\partial I_{2}^{+}$and requires the exploitation of (3.8). The derivation of a general expression in terms of the principal solution and the eigenmode for the discontinuity terms in (2.37) is not at all easy as this relatively simple example indicates. The independent derivation of (3.14) using the general approach developed in the previous section provides an additional check for the results obtained in this example.

\section{Conclusions}

In the present work, the general bifurcation and postbifurcation analysis for generalized standard continua has been presented.

Adopting the generalized standard material formalism yields certain advantages over Hill's classical formulation of the problem. More specifically, the issue of bifurcations of rate higher than one can be addressed in a straightforward manner. Hill's bifurcation exclusion criterion is found to be more general than had been proved thus far, since it is valid for bifurcations of any rate. In addition, a consistent methodology that takes properly into account all the field quantities discontinuities, is given for the postbifurcation expansions in boundary value problems, without the restrictions of total loading in the principal branch of the solution which have been required previously.

The theory, in its present form, is applicable to small strain and moderate rotations of rate independent elastoplastic solids obeying the maximum dissipation principle. 
The reasons for this limitation is the lack (at least up to the present) of a satisfactory generalization of the generalized standard material formulation for the case of finite strains. For this case, the Hill-Hutchinson approach still remains the only method for analysing the angular bifurcation and postbifurcation problem. Moreover, the smooth bifurcation and postbifurcation issue (always in the large strain context) has only been addressed via the use of a Christoffersen-Hutchinson type model as discussed by Triantafyllidis (1983), an approach that is limited to a particular class of constitutive laws that are very difficult to verify experimentally. Once an acceptable generalized standard material formalism can be found for rate-independent materials in the large strain regime, the present bifurcation and postbifurcation analysis can easily be extended using essentially the same approach.

\section{ACKNOWLEDGEMENTS}

One of us (N.T.) would like to acknowledge the constructive criticism of Professor R. HILL, which in essence was the motivation for the present work. This work was completed while one of us (N.T.) was visiting with the Fcole Polytechnique during the Fall of 1985. The warm hospitality of the Solid Mechanics Lab. of the School is gratefully appreciated.

\section{REFERENCES}

BUDIANSKY, B.

Christoffersen, $\mathrm{J}$, and HUTCHINSON, J. W.

CONSIDERE, A.

HAlphen, B. and NGUYen, Q. S.

HILL, R.

HILL, R.

HutChinson, J. W.

Hutchinson, J. W.

KOITER, W. T.

NGUYEN, Q. S.

NGUYEN, Q. S.

NGUyen, Q. S.

Nguyen, Q. S. and Stolz, C.

Petryk, $H$. and Thermann, $K$.

Shanley, F. R.

TRIANTAFYLlidis, N.

VON Karman, T.
1974 Adc. Appl. Mech. 14, 1.

1979 J. Mech. Phys. Solids 27, 465.

1891 Cong. Intl Proc. Const. p. 371, Paris.

1975 J. Mécanique 14, 39.

1956 J. Mech. Phys. Solids 4, 247.

1958 J. Mech. Phys. Solids 6, 236.

1973 J. Mech. Phys. Solids 21, 163.

1974 Adr. Appl. Mech. 14, 67.

1945 Doctoral Thesis, Delft.

1973 Doctoral Thesis, Paris.

1980 Problèmes de Plasticité et de Rupture Course Notes. Univ. of Orsay.

1987 J. Mech. Phys. Solids 35, 123.

1985 C. R. Acad. Sci. Paris, V.300, Ser. II, No. 7, p. 235.

1985 J. Mech. Phys. Solids 33, 577.

1947 J. Aeronaut. Sci. 14, 261.

1983 J. Mech. Phys. Solids 31, 499.

1910 Verein Deutcher Ingenieure, Vol. 81. 